predominated (Millichap JG, Dodge PR. Neurology 1960; 10:1007). The authors postulated that the facial abnormalities were secondary to the neuromuscular defect. Congenital myasthenia has been described in association with arthrogryposis, the subject of the following article.

\title{
ARTHROGRYPOSIS CONGENITA AND HEPATORENAL ABNORMALITIES
}

Arthrogryposis multiplex congenita with renal and hepatic abnormalities, demonstrated at autopsy in a two month old child of consanguineous parents, is reported from the Pediatric Hospital, Coimbra, Portugal. Three brothers and eight first cousins had died within the first month, all with jaundice. The brothers of the proband had limb abnormalities and one had polyuria, glucosuria, and metabolic acidosis. The patient was born with flexed knees and joint limitation, cubital deviation of the hands with clenched fingers, and muscular atrophy. During the second week of life the infant became jaundiced and on day 18 she was admitted with cholestatic jaundice and hepatomegaly. Electromyography and muscle biopsy were compatible with neuropathic muscular atrophy. There was hypercalcemia with increased density of the base of the skull, renal tubular degeneration, and biliary stasis with pigmentary deposits. The family pedigree suggested an autosomal recessive inheritance. (Saraiva JM et al. Arthrogryposis multiplex congenita with renal and hepatic abnormalities in a female infant. J Pediatr Nov 1990; 117:761-763).

COMMENT. This syndrome was first described by Nezelhof C et al (J Pediatr 1979; 94:258) who reported four patients with these findings. As found in this case report, arthrogryposis is most commonly associated with neuropathic muscular atrophy. The underlying lesion may be found in the anterior horn cells of the spinal cord, the peripheral nerves, the neuromyal junction, the muscle, and sometimes in the brain.

\section{INFECTIOUS DISEASE}

NEUROLOGIC MANIFESTATIONS OF LYME DISEASE

The chronic neurologic symptoms and signs in 27 patients with previous signs of Lyme disease and current evidence of immunity to Borrelia burgdorferi are reported from the Departments of Neurology and Medicine, Tufts University School of Medicine, New England Medical Center, Boston, MA. The median age was 49 years with a range of 25-72 years. Signs and symptoms of chronic neurologic abnormalities included encephalopathy in $89 \%$ with memory loss, depression, sleep disturbance, irritability, and difficulty finding words; polyneuropathy in $70 \%$ with spinal or radicular pain, distal paresthesia, and sensory loss; leukoencephalitis in $4 \frac{\circ}{5}$; and miscellaneous symptoms including fatigue $(74 \%)$, headache $(48 \%)$, hearing loss (15\%), fibromyalgia (15\%), and tinnitus $\left(7 \frac{\circ}{0}\right)$. Seventeen patients $(63 \%)$ had abnormalities of both the central and peripheral nervous systems, seven (26\%) had encephalopathy alone, two ( $7 \%$ ) had polyneuropathy alone, and the remaining patient 
(4\%) had leukoencephalitis. At the time of examination chronic neurologic abnormalities had been present from three months to 14 years, usually with little progression. Six months after a two week course of intravenous Ceftriaxine ( $2 \mathrm{~g}$ daily), 17 patients (63\%) had improved, 6 (22\%) had improved and then relapsed, and 4 (15\%) had no change in their condition. The early signs of infection were erythema migrans ( $85 \%)$, and headache and neck st iffness in $41 \%$. The most common early neurologic abnormality was a facial palsy in $30 \%$. Arthritis had occurred in $70 \%$ before the chronic neurologic symptams developed and it was still present in ten patients (37\%) when the chronic neurologic abnormalities were noted. (Logigian EL et al. Chronic neurologic manifestations of Lyme disease). $\underline{N}$ Engl J Med Nov 22, 1990; 323: $1438-44)$.

COMMENT. The criteria for case inclusion in this study were previous signs of Lyme disease, neurologic symptoms lasting at least three months that could not be attributed to another cause, and current evidence of humoral or cellular immity to B. burgdorferi, as shown by elevated serum IgG or IgM antibody titer of at least 1:400, five or more IgG antibody bands to spirochetal polypeptides, or a stimulation index of 10 or more in response to Borrelia antigens. The chronic neurologic abnormalities began months to years after the onset of infection, sometimes after long periods of latency and similar to the course of neurosyphilis. The most common form of chronic aNS involvement was subacute encephalopathy affecting memory, mood, and sleep sometimes with subtle disturbances in language. Diagnosis could be difficult because of the nonspecific nature of the symptams. Although this report concerns adults, the findings might be pertinent to Lyme disease contracted during childhood.

\section{MUSCLE DISEASE}

\section{DIAGNOSIS OF POLYMYOSITIS}

The criteria for the diagnosis of polymyositis, the differential diagnosis, and treatment are reported from the Division of Rheumatology and Internal Medicine, Mayo Clinic, Rochester, MN. A muscle biopsy is considered the standard diagnostic procedure for polymyositis but it is not infallible. Characteristic features are inflammatory mononuclear cell infiltration, degenerating and regenerating muscle fibers, and central nuclei. Electromyographic findings are characteristic but not specific. They include insertional activity, fibrillation potentials, motor unit potentials of increased frequency and decreased duration, and normal conduction velocity in nerves. The most useful blood test in polymyositis is a serum CK determination but this may be normal in about $2 \frac{\mathrm{o}}{\mathrm{r}}$ of patients. The MM fraction constitutes the majority of the $\mathrm{CK}$ in polymyositis, the $\mathrm{MB}$ fraction may be increased because of skeletal muscle regeneration and less often because of myocarditis. Treatment with high dose corticosteroids is administered as early as 